\title{
PREVALENCE OF PERIODONTAL DISEASE IN WOMEN WITH POLYCYSTIC OVARY SYNDROME- A COMPARATIVE DESCRIPTIVE STUDY
}

\author{
Sai Darshana Nair1, Siddhartha Varma², Girish Suragimath³, Sameer Zope ${ }^{4}$, Vishwajeet Kale ${ }^{5}$, Keshava Abbayya ${ }^{6}$ \\ ${ }_{1}^{1}$ Postgraduate Student, Department of Periodontology, School of Dental Sciences, Krishna Institute of Medical Science Deemed \\ University. \\ ${ }^{2}$ Reader, Department of Periodontology, School of Dental Sciences, Krishna Institute of Medical Science Deemed University. \\ ${ }^{3}$ HOD, Department of Periodontology, School of Dental Sciences, Krishna Institute of Medical Science Deemed University. \\ ${ }^{4}$ Senior Lecturer, Department of Periodontology, School of Dental Sciences, Krishna Institute of Medical Science Deemed University. \\ ${ }^{5}$ Senior lecturer, Department of Periodontology, School of Dental Sciences, Krishna Institute of Medical Science Deemed University. \\ ${ }^{6}$ Reader, Department of Periodontology, School of Dental Sciences, Krishna Institute of Medical Science Deemed University.
}

ABSTRACT

\section{BACKGROUND}

Polycystic ovary syndrome (PCOS) is a common disorder affecting $4 \%$ to $12 \%$ of women of reproductive age. Increased production of steroid hormones is associated with increased gingival inflammation. Thus, the present study was aimed to determine the association between periodontal diseases and polycystic ovary syndrome.

\section{MATERIALS AND METHODS}

A total of 240 women of age group between 18-45 years were enrolled into the study by convenient sampling technique. Since the duration of the study was 3 months, only 120 PCOS patients had reported to the Department of Gynaecology. Thus, we had enrolled 120 women with PCOS and 120 women without PCOS into this study in order to compare the periodontal status of both groups. PCOS diagnosis was made according to the Rotterdam criteria. Periodontal status for both the above said groups were evaluated using Gingival index (GI) and Clinical attachment level (CAL). Data were analysed using Mann-Whitney U test \& Fisher's exact test. Statistical significance was defined at $\mathrm{P}<0.05$.

\section{RESULTS}

CAL, body mass index and GI were significantly higher in women with PCOS compared to women without PCOS.

\section{CONCLUSION}

The prevalence of periodontal disease seems to be higher in women with PCOS. This may be attributed to the role of chronic systemic inflammation that contributes to the pathophysiology of PCOS and periodontal disease.

\section{KEYWORDS}

Gingivitis, Hormones, Periodontitis.

HOW TO CITE THIS ARTICLE: Nair SD, Varma S, Suragimath G, et al. Prevalence of periodontal disease in women with polycystic ovary syndrome- A comparative descriptive study. J. Evolution Med. Dent. Sci. 2017;6(65):4733-4736, D0I: $10.14260 /$ Jemds/2017/1025

\section{BACKGROUND}

Polycystic ovary syndrome (PCOS) also known as SteinLeventhal Syndrome is a common endocrine disorder seen in $4-12 \%$ of women of childbearing age. It develops shortly after puberty and causes accumulation of undeveloped follicles in ovaries which later becomes a cyst.[1,2] PCOS is a common disorder with a significant public health impact. Reproductive derangements, other metabolic and psychological comorbidities are common findings in women with PCOS.[3] Apart from menstrual abnormalities (Oligomenorrhoea or amenorrhea) and ultrasound findings (Polycystic ovaries), PCOS also presents with clinical/biochemical evidence of hyperandrogenism

Financial or Other, Competing Interest: None.

Submission 10-07-2017, Peer Review 03-08-2017,

Acceptance 09-08-2017, Published 14-08-2017.

Corresponding Author:

Dr. Sai Darshana Nair,

Building B1, Apartment 12,

Krishna Institute of Medical Science Deemed University,

Malkapur, Karad-District, Satara-415539,

Maharashtra, India.

E-mail: saidarshana1@gmail.com

DOI: $10.14260 /$ jemds $/ 2017 / 1025$

\section{(c) (i) $\ominus$}

(Hirsutism, acne, or androgenic alopecia).[4] Women with PCOS display an adverse cardiometabolic risk profile, including insulin resistance(IR), central obesity, dyslipidaemia and increased prevalence of cardiovascular risk factors. Thus, PCOS can be regarded as a gender-specific form of the metabolic syndrome.

Periodontitis is a chronic inflammatory disease of the tooth supporting structures, triggered against pathogenic bacterial microflora. The subsequent exuberant inflammatory response leads to alveolar bone loss resulting in loss of tooth. Several literatures have already evidenced the association between periodontitis and systemic disorders, including metabolic syndrome, diabetes, and cardiovascular disease (CVD).[5-9] The fact that both periodontal disease and metabolic syndrome are associated with systemic inflammation and IR, these two disorders may be linked through a common pathophysiological pathway. Literature suggests that the role of oxidative stress could be the pertinent factor for the association between periodontal diseases and components of the metabolic syndrome, diabetes, and CVD. Females with PCOS have increased markers of lipid peroxidation, elevated levels of C-reactive protein, inflammatory cytokines, as well as higher percentage of blood lymphocytes and monocytes. ${ }^{[9-11]}$ 
Growing evidence supports the concept that PCOS is associated with increased oxidative stress and systemic inflammation. ${ }^{[12-13]}$ An increased oxidative stress may in turn contribute to insulin resistance, ovarian dysfunction, and other characteristics of PCOS. Taking the above said factors in to consideration, the present study was aimed to compare the periodontal status of women with and without PCOS to explore the impact of PCOS on the progression of periodontal disease.

\section{MATERIALS AND METHODS}

The current descriptive comparative study was undertaken in the Department of Gynaecology, Krishna Institute of Medical Sciences, Karad. Ethical clearance was obtained before commencing the study (Ref. No. KIMSDU/IEC/02/2015). The duration of this study was three months that extended from April 2015 to June 2015 in which a total of 240 women were enrolled into the study by convenient sampling technique after obtaining their due consent. Out of 240 women, 120 women comprised of the PCOS group. The diagnosis of PCOS was arrived based on history, clinical signs, physical examination, laboratory parameters, and ultrasound findings, as per Rotterdam criteria[4] (Table I) by the Gynaecologist. As the duration of the study was short (3 months), there were only 120 PCOS patients who had reported to the Department of Gynaecology in that time span. Thus, same number of age matched systemically healthy women without PCOS were enrolled as healthy controls $(n=120)$. Thus, sample size estimation was also done at convenience.

\section{Inclusion Criteria}

Women in the age range of 18-45 years having minimum of 20 teeth were included. PCOS group comprised of 120 women who were diagnosed with PCOS as per Rotterdam criteria and 120 healthy controls without PCOS and who were systemically healthy.

\section{Exclusion Criteria}

Pregnant women, smokers, alcoholics, women suffering from malignancy or osteoporosis were excluded. Women who had undergone periodontal treatment or those women who was under prophylactic antibiotic therapy during the past six months were excluded from the study.

\section{Periodontal Examination}

A single calibrated examiner (SD) carried out the periodontal examination of all the 240 women who were enrolled into the study under the supervision of a senior periodontist (SV) to minimise the observer bias. The periodontal disease severity was assessed using periodontal parameters like gingival index (GI) and clinical attachment loss (CAL).

All women included in the study were subjected to periodontal examination in the early follicular phase which is mainly seen in $1^{\text {st }}$ to $13^{\text {th }}$ day of the last menstrual cycle. This was done to minimise the effects of hormonal changes during menstrual cycle. ${ }^{[14-17]}$ The GI and CAL were calculated at four sites on each tooth. The GI was assessed after drying gingiva with cotton rolls.[18] The tissue surrounding each tooth were divided into four gingival scoring units (Distofacial papilla, mesiofacial papilla, facial margin and lingual gingival margin). A Williams periodontal probe (Hu-Friedy) was used to measure CAL. The probe is inserted parallel to the vertical axis of the tooth and walked circumferentially around each surface of the tooth. The measurements for CAL were recorded at four sites per tooth (Mesiobuccal, distobuccal, midbuccal and midlingual) of all teeth except third molars. CAL was measured from cementoenamel junction to the base of the gingival sulcus. The women were classified as having chronic periodontitis based on American Academy of Periodontology 1999 consensus classification of periodontal diseases.[19] Chronic periodontitis patients were characterised into three categories depending on level of average clinical attachment loss, slight: 1-2 mm CAL, moderate: 3-4 mm CAL and severe: $\geq 5 \mathrm{~mm}$ CAL. Body mass index (BMI) of all the women were calculated by dividing weight in kilogram upon height in metre square and tabulated. Commonly accepted BMI ranges are: underweight: under $18.5 \mathrm{~kg} / \mathrm{m}^{2}$, normal weight: 18.5 to 25 , overweight: 25 to 30 , obese: over 30 .

\section{Statistical Analysis}

Statistical analysis was carried out using SPSS software version 20.0 (SPSS Inc., Chicago, IL, USA). Mann-Whitney U test and Fisher's exact test were used for the comparison of values between two groups. P value $<0.05$ was considered as statistically significant.

\section{RESULTS}

The mean age in PCOS group and healthy group were $23.6 \pm$ 4.46 and $23.40 \pm 4.47$ years respectively. Clinical characteristics and periodontal parameters of both groups are summarised in Table II. BMI, CAL and GI were statistically significant between both groups with a $\mathrm{p}$ value less than $<0.05$. Table III shows the periodontal disease severity in PCOS and healthy group. There was statistically significant difference in the prevalence of periodontal disease in PCOS group as compared to healthy group with a $\mathrm{P}$ value of $<0.05$.

Rotterdam Criteria $2004^{4}$

Exclusion of other androgen excess or related disorders Includes two of the following:

- Clinical and/or biochemical hyperandrogenism

- Oligo-ovulation or anovulation

- Polycystic ovaries

Table I. Rotterdam Criteria for the Diagnosis of Polycystic Ovarian Syndrome

\begin{tabular}{|c|c|c|c|c|c|}
\hline Parameters & \multicolumn{2}{|c|}{ PCOS Group (n=120) } & \multicolumn{2}{c|}{ Healthy Group (n=120) } & P \\
\hline & Mean \pm SD & $\begin{array}{c}\text { 95\% CI } \\
\text { (Lower- Upper Bound) }\end{array}$ & Mean \pm SD & $\begin{array}{c}\text { 95\% CI } \\
\text { (Lower- Upper Bound) }\end{array}$ & 0.651 \\
\hline Age & $23.40 \pm 4.47$ & $21.73-25.07$ & $23.6 \pm 4.46$ & $21.93-25.27$ & $0.034^{*}$ \\
\hline BMI & $24.39 \pm 4.52$ & $22.7-26.07$ & $22.09 \pm 3.81$ & $20.67-23.51$ & $0.046^{*}$ \\
\hline CAL & $1.28 \pm 1.31$ & $0.79-1.77$ & $0.57 \pm 0.73$ & $0.29-0.84$ & $<0.001^{*}$ \\
\hline GI & $2.13 \pm 0.26$ & $2.04-2.23$ & $1.12 \pm 0.12$ & $1.07-1.16$ & \\
\hline \multicolumn{2}{|r}{ Table II. Comparison of Clinical Characteristics and Periodontal Parameters between PCOS Group and } \\
\end{tabular}

* Statistical significance

Data are presented as mean \pm standard deviation. 


\section{Abbreviations}

SD- standard deviation; CI- confidence interval; BMI- body mass index; CAL- clinical attachment level; GI- gingival index.

\begin{tabular}{|c|c|c|c|c|c|}
\hline Severity & $\begin{array}{c}\text { PCOS } \\
\text { Group } \\
\text { (n) }\end{array}$ & \% & $\begin{array}{c}\text { Healthy } \\
\text { Group } \\
\text { (n) }\end{array}$ & \% & P \\
\hline Mild & 0 & 0.00 & 56 & 46.67 & \multirow{2}{*}{$<0.001^{*}$} \\
\hline Moderate & 44 & 36.67 & 64 & 53.33 & \\
\hline Severe & 76 & 63.33 & 0 & 0.00 & \\
\cline { 1 - 4 } & & &
\end{tabular}

Table III. Periodontal Disease Severity in PCOS Group and Healthy Group Assessed using Fisher's Exact Test

Slight: 1-2 mm of attachment loss (AL), Moderate: 3-4 mm of AL, Severe $>5 \mathrm{~mm}$ of AL.

\section{DISCUSSION}

PCOS is a complex endocrinal disorder that manifests with a wide range of variations in clinical manifestations. It is impossible to pinpoint out a single causative factor that is responsible for the clinical manifestation of this condition. [20] A higher prevalence of periodontal disease was observed in women with PCOS compared to women without PCOS (Healthy controls) in this study, though the participants in both the groups were matched in terms of possible confounding variables such as age and BMI. Similar findings were observed in a study conducted by Dursun et al[21] where women with PCOS showed higher periodontal indices. It is a well-documented fact that hormonal changes occurring during puberty, pregnancy, and menstrual cycles have an impact over the gingival health of women. ${ }^{[22]}$ In PCOS women, ovulation and follicle growth often do not occur due to the disrupted levels of follicular stimulating hormone (FSH) and luteinising hormone (LH). Extra LH production leads to the excessive production of androgens. This excess androgen is responsible for the many features like hirsutism, acne and weight gain. It is a known fact that the increase in the steroid hormone levels like oestrogen causes gingival inflammation.

The hyperandrogenism status commonly seen in PCOS patients is responsible for the menstrual abnormalities and infertility in such women. The increase in steroid levels in women with PCOS alters the gingival response to microbial challenge. The gingiva often becomes hyperresponsive to mildest microbial deposits, leading to greater periodontal destruction, making such patients more vulnerable to periodontal destruction. Gingival tissues respond to the hormonal level alteration by initiating changes in oral flora and also by the increased production of pro-inflammatory cytokines. In turn, these changes adversely affect the bones and adhesive joints eventually leading to tooth loss.[23] Literature suggests that oxidative stress which is commonly seen in PCOS patients may be a common link for the association between periodontitis and components of the metabolic syndrome, diabetes, and CVD.[12] A statistically significant difference was noticed in terms of BMI, CAL and GI levels between women with PCOS and without PCOS. Obesity is a common finding in women with PCOS due to glucose intolerance. A metabolic derangement in which the aromatase enzyme usually seen in the adipose tissue, converts the testosterone to oestrogen in PCOS patients. As a result of excess oestrogen production, a vicious cycle of intense hormonal effects on periodontal tissues is triggered.
The higher BOP rates in PCOS group were attributed to the influence of hyperandrogenism on the vascular flora as observed in other studies.[17] Increased CAL in PCOS group is due to the hyperresponsive inflammatory process that is produced as a result of hormonal imbalance. These inflammatory processes play an imperative role in the progression of periodontal disease and subsequent increase in the gingival sulcus depth and bone loss. However, the frequency of periodontitis in women with and without PCOS was not statistically significant in a recent study conducted by Porwal et al[23] which is contradictory to our study where in moderate periodontitis was higher in PCOS group compared to healthy control group without PCOS. These findings need further confirmation in future studies, with large sample size to elucidate any underlying relationships between PCOS and periodontitis.

\section{Limitation of the Study}

Due to the short duration of the study, convenience sampling technique was followed. Thus, sampling size was also calculated by convenience. The results of this study cannot be generalised due to the potential bias resulting from the sampling technique and sample size estimation.

\section{CONCLUSION}

Within the limitations of this study, a higher prevalence of periodontal disease parameters was observed in the PCOS group when compared to the women without PCOS. Thus, PCOS patients might have a greater likelihood for the development of more severe forms of periodontitis at a later date.

\section{Acknowledgements}

We are thankful to the Department of Gynaecology, Krishna Institute of Medical Sciences, Karad for their support in conducting this project.

\section{REFERENCES}

[1] Knochenhauer ES, Key TJ, Kahsar-Miller M, et al. Prevalence of the polycystic ovary syndrome in unselected black and white women of the southeastern United States: a prospective study. J Clin Endocrinol Metab 1998;83(9):3078-82.

[2] Farah L, Lazenby AJ, Boots LR, et al. Prevalence of polycystic ovary syndrome in women seeking treatment from community electrologists. Alabama professional electrology association study group. J Reprod Med 1999;44(10):870-4.

[3] Rahiminejad ME, Moaddab A, Rabiee R, et al. The relationship between clinicobiochemical markers and depression in patient with polycystic ovary syndrome. Iran J Reprod Med 2014;12:811-6.

[4] Rotterdam ESHRE/ASRM-Sponsored PCOS Consensus Workshop Group. Revised 2003 consensus on diagnostic criteria and long-term health risks related to polycystic ovary syndrome. Fertil Steril 2004;81(1):19-25.

[5] Katz J, Flugelman MY, Goldberg A, et al. Association between periodontal pockets and elevated cholesterol and low density lipoprotein cholesterol levels. J Periodontol 2002;73(5):494-500. 
[6] Reeves AF, Rees JM, Schiff M, et al. Total body weight and waist circumference associated with chronic periodontitis among adolescents in the United States. Arch Pediatr Adolesc Med 2006;160(9):894-9.

[7] Genco RJ, Grossi SG, Ho A, et al. A proposed model linking inflammation to obesity, diabetes and periodontal infections. J Periodontol 2005;76(11 Suppl):2075-84.

[8] Kim J, Amar S. Periodontal disease and systemic conditions: a bidirectional relationship. Odontology 2006;94(1):10-21.

[9] Marchetti E, Monaco A, Procaccini L, et al. Periodontal disease: the influence of metabolic syndrome. Nutr Metab (Lond) 2012;9:88.

[10] Liu Z, Liu Y, Song Y, et al. Systemic oxidative stress biomarkers in chronic periodontitis: a meta-analysis. Dis Markers 2014;2014:10.

[11] Duleba AJ, Dokras A. Is PCOS an inflammatory process? Fertil Steril 2012;97(1):7-12.

[12] Bullon P, Morillo JM, Ramirez-Tortosa MC, et al. Metabolic syndrome and periodontitis: is oxidative stress a common link? J Dent Res 2009;88(6):503-18.

[13] Murri M, Luque-Ramírez M, Insenser $M$, et al. Circulating markers of oxidative stress and polycystic ovary syndrome (PCOS): a systematic review and meta-analysis. Hum Reprod Update 2013;19(3): 268-88.

[14] Chaffee BW, Weston SJ. Association between chronic periodontal disease and obesity: a systematic review and meta-analysis. J Periodontol 2010;81(12): 1708-24.
[15] Sharland G, Rollings S, Simpson J, et al. Hypoplastic left-heart syndrome. Lancet 2001;357(9257):722.

[16] Koreeda N, Iwano Y, Kishida M, et al. Periodic exacerbation of gingival inflammation during the menstrual cycle. J Oral Sci 2005;47(3):159-64.

[17] Machtei EE, Mahler D, Sanduri H, et al. The effect of menstrual cycle on periodontal health. J Periodontol 2004;75(3):408-12.

[18] Shourie V, Dwarakanath CD, Prashanth GV, et al. The effect of menstrual cycle on periodontal health-a clinical and microbiological study. Oral Health Prev Dent 2012;10(2):185-92.

[19] Armitage GC. Development of a classification system for periodontal diseases and conditions. Ann Periodontol 1999;4(1):1-6.

[20] Asmathulla S, Kripa S, Rajarajeswari R, et al. Insulin resistance and its relation to inflammatory status and serum lipids among young women with PCOS. International Journal of Reproduction, Contraception, Obstetrics and Gynecology 2016;2(3):325-9.

[21] Dursun E, Akalin FA, Güncü GN, et al. Periodontal disease in polycystic ovary syndrome. Fertil Steril 2011;95(1):320-3.

[22] Butler BL, Morejon O, Low SB. An accurate, timeefficient method to assess plaque accumulation. J Am Dent Assoc 1996;127(12):1763-6.

[23] Porwal S, Tewari S, Sharma RK, et al. Periodontal status and high-sensitivity C-reactive protein levels in polycystic ovary syndrome with and without medical treatment. J Periodontol 2014;85(10):1380-9. 